\title{
ACCURATE MASSES AND RADII AS TEST DATA FOR STELLAR MODELS: STATUS AND PROGRESS ON THE COPENHAGEN PROJECT
}

J. ANDERSEN, J.V. CLAUSEN, B.E. HELT, K.T. JOHANSEN and

B. NORDSTRÖM, Copenhagen University Observatory, Denmark

M. GARCÍA, Departamento de Física, E.I. Industriales, Madrid, Spain

A. GIMÉNEZ, Laboratorio de Física Espacial, INTA, Madrid, Spain

R.P. STEFANIK, Harvard-Smithsonian Center for Astrophysics, U.S.A.

L.P.R. VAZ, Departamento de Fisica, Univ. Fed. de Minas Gerais, Brazil

\section{Basic ingredients of stellar models}

The most important ingredient of a stellar model is its initial mass. Hence, real stars with known masses are important test objects for stellar models. Initial chemical composition is the other important starting parameter and should therefore also be known (Clausen, 1992). Finally, the most direct indicator of the evolution of a star is its radius. Together, these parameters determine the effective temperature and luminosity of the model, to be tested against a real star.

\section{How accurate need the data be?}

For such comparisons to give interesting results on present-day stellar models, the empirical data must be accurate $\left(1-2 \%\right.$ in $M, R$, and $T_{e} ; 0.1$ dex in [Fe/H]): Stellar masses with errors of just $\pm 5 \%$ can yield essentially no useful information on modern (near-main-sequence) stellar models.

\section{What exists, and what more is needed?}

Data of the required accuracy can presently only be obtained from selected, detached, double-lined eclipsing binaries. The current status of such data (Andersen, 1991) is summarized in Fig. 1. Here, the basic data $M$ and $R$ are displayed in a format designed to bring out the effects of evolution through the main-sequence band (note the size of the error bar!) as defined by (dashed) standard or (dots) overshooting models (Claret \& Gimenez, 1991). Data obtained within the framework of the Copenhagen (CUO) program are identified. 


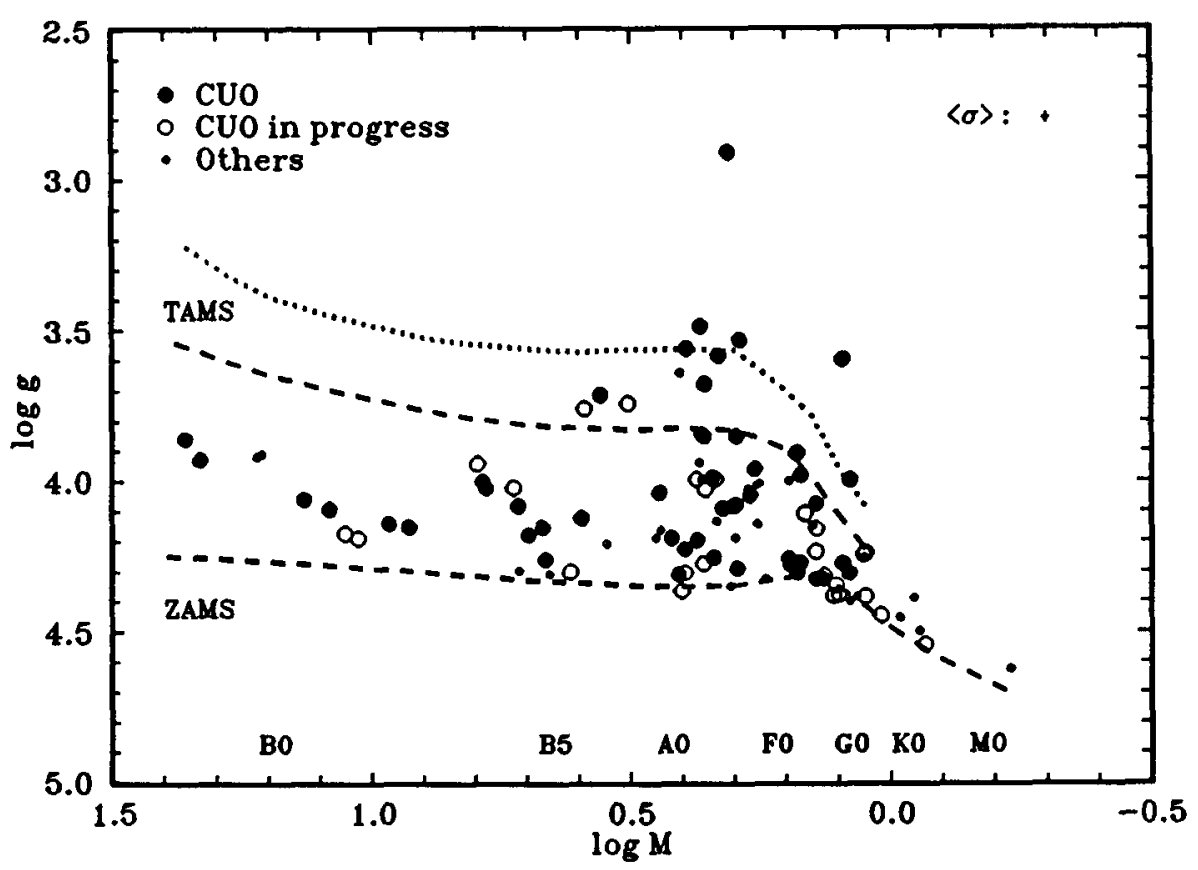

Fig. 1. $\log M-\log g$ diagram for binary components with mass and radius known to $\pm 2 \%$ or better.

Systems now in progress (open circles) are primarily:

- Early-type (OB) stars,

- Stars useful in locating the empirical ZAMS,

- Stars with $[\mathrm{Fe} / \mathrm{H}]$ determination in progress, useful in testing opacity calculations.

\section{The observational basis}

As an example of the quality of the observational data needed to produce masses and radii which are useful for our purposes, preliminary light and radial-velocity curves for one of the new systems are shown in Figs. 2a,b.

\section{References}

Andersen, J. 1991, Astr. Ap. Rev., 3, 91.

Clausen, J.V. 1992, in Inside the Stars, eds. W. Weiss and A. Baglin.

Claret, A., Giménez, A. 1991, Astr. Ap. Suppl., 87, 507. 


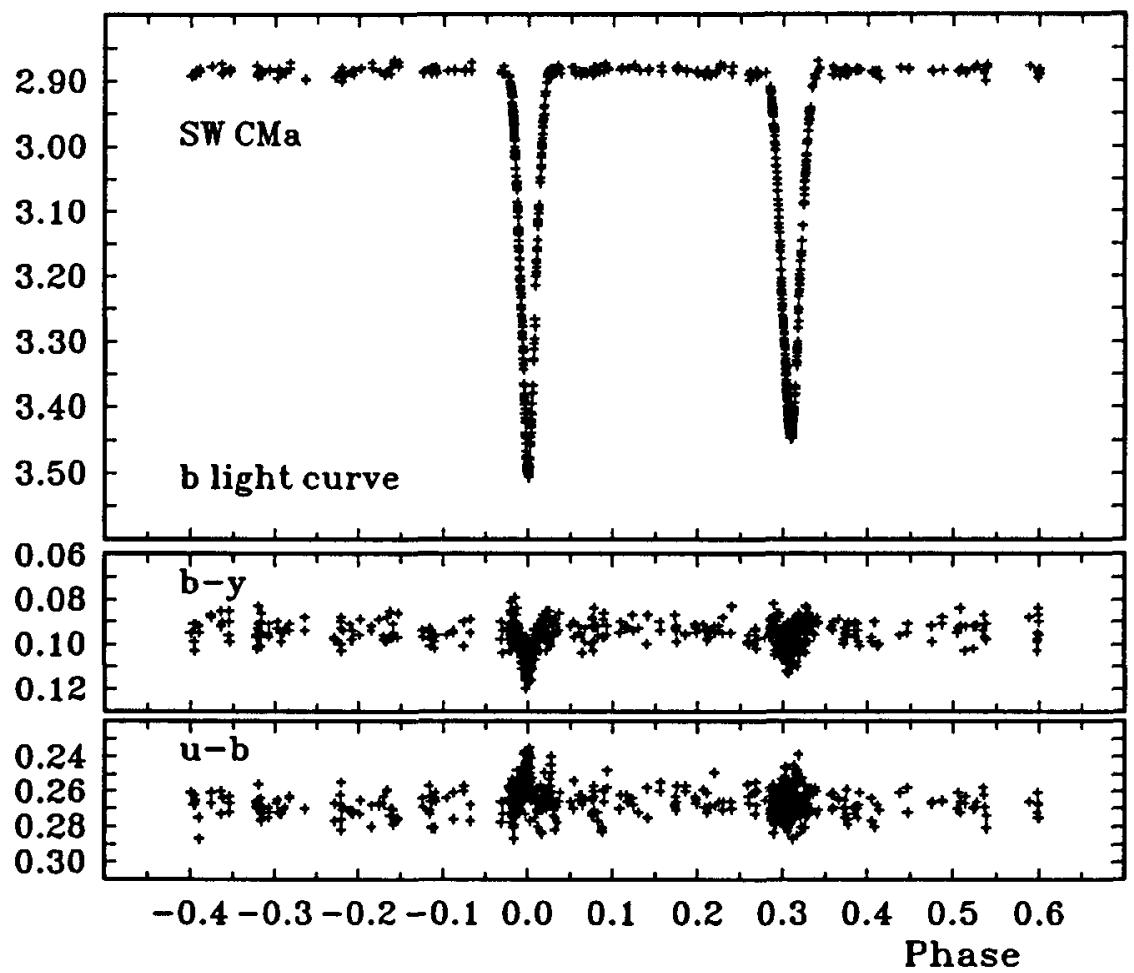

Fig. 2a. Light and colour curves of SW CMa (Danish 50-cm tel., ESO, La Silla).

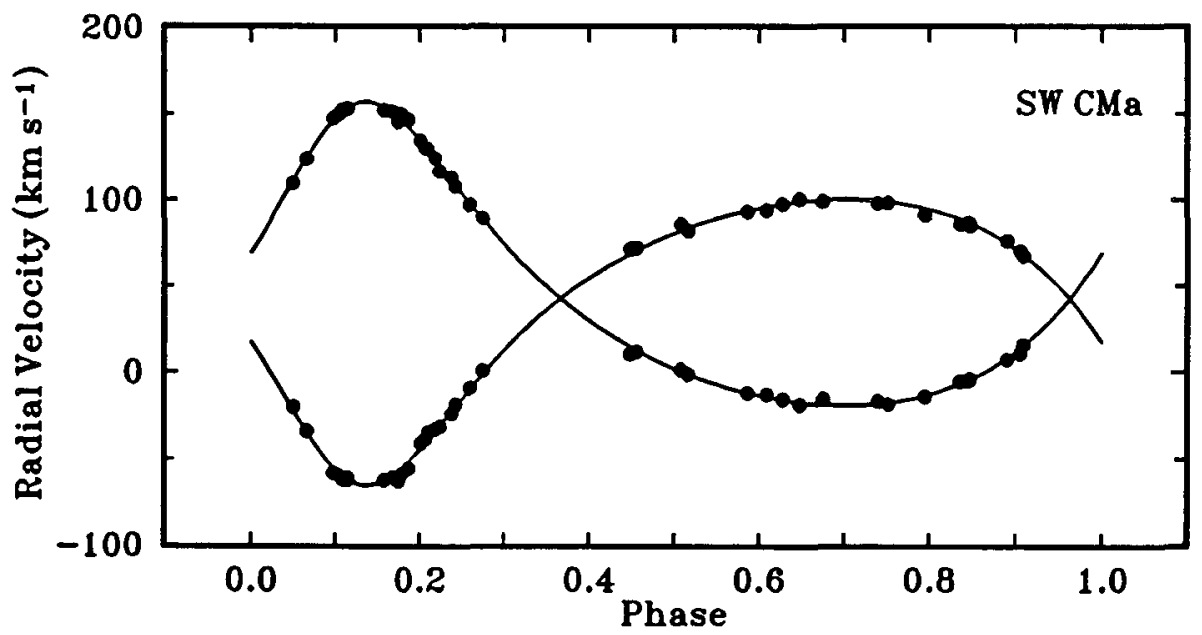

Fig. 2b. Radial-velocity curves of SW CMa (CfA echelle systems). 